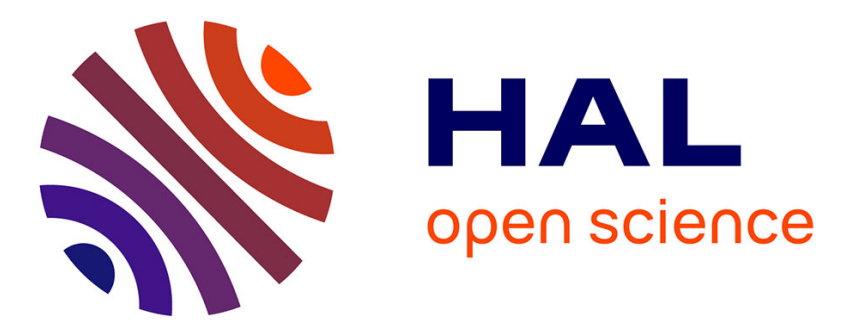

\title{
A new approach for volume reconstruction in TomoPIV with the alternating direction method of multipliers
}

\author{
Ioana Barbu, Cédric Herzet
}

\section{To cite this version:}

Ioana Barbu, Cédric Herzet. A new approach for volume reconstruction in TomoPIV with the alternating direction method of multipliers. Measurement Science and Technology, 2016, 27 (10), pp.21. 10.1088/0957-0233/27/10/104002 . hal-01419329

\section{HAL Id: hal-01419329 \\ https://hal.inria.fr/hal-01419329}

Submitted on 19 Dec 2016

HAL is a multi-disciplinary open access archive for the deposit and dissemination of scientific research documents, whether they are published or not. The documents may come from teaching and research institutions in France or abroad, or from public or private research centers.
L'archive ouverte pluridisciplinaire HAL, est destinée au dépôt et à la diffusion de documents scientifiques de niveau recherche, publiés ou non, émanant des établissements d'enseignement et de recherche français ou étrangers, des laboratoires publics ou privés. 


\title{
A New Approach for Volume Reconstruction in TomoPIV with the Alternating Direction Method of Multipliers
}

\author{
Ioana Barbu ${ }^{1}$ and Cédric Herzet ${ }^{2}$ \\ ${ }^{1}$ ACTA, Irstea Centre Rennes, France \\ ${ }^{2}$ Fluminance, INRIA Centre Rennes - Bretagne Atlantique, France \\ E-mail: ${ }^{1}$ ioana.barbu@irstea.fr,${ }^{2}$ cedric.herzet@inria.fr
}

\begin{abstract}
We adapt and import into the TomoPIV scenery a fast algorithm for solving the volume reconstruction problem. Our approach is based on the reformulation of the volume reconstruction task as a constrained optimization problem and the resort to the "Alternating Directions Method of Multipliers" (ADMM). The inherent primaldual algorithm is summarized in this article to solve the optimization problem related to the TomoPIV. In particular, the general formulation of the volume reconstruction problem considered in this paper allows to: $i$ ) take explicitly into account the level of the noise affecting the data; ii) account for both the nonnegativity and the sparsity of the solution. Experiments on a numerical TomoPIV benchmark show that the proposed framework is a serious contender for the state-of-the-art.
\end{abstract}

Keywords: TomoPIV, Volume reconstruction, Optimization

Submitted to: Meas. Sci. Technol.

\section{Introduction}

Fluid visualization systems have become a pervasive means of understanding the tridimensional (3D) motion of turbulent flows. Among the most popular, the "Particle Image Velocimetry" (PIV) [26] consists in tracking the bidimensional (2D) motion of lightly seeded particles immersed in the flow and visible within a thin laser sheet. Per contra, the technique - coupled with optical flow estimation algorithms - fails to detect highly 3D vortical structures inherent to turbulence. Recently, PIV has shifted towards a 3D setting. The so-called "Tomographic PIV"(TomoPIV) system, introduced by Elsinga et al in [19], enables the inference of the 3D motion of a flow from the observation of a multiview sequence of images capturing the 3D scene.

A crucial step in the development of TomoPIV is the volumetric reconstruction of the particles' distribution at each time frame, see [4, Chapter 3] for a review. The volume reconstruction problem is based on the inversion of a linear model connecting 
the TomoPIV signal (i.e., the 3D distribution of particles at each time frame) to the set of multiview images. Unfortunately, this system of equations is intrinsically underdetermined and of very high dimensionality. This leads to two major problems. First, as a consequence of its underdetermination, the system to invert has usually an infinity of solutions; one should therefore exploit some additional knowledge on the signal of interest to single out a proper solution. Second, the very high dimensionality of the system may render its inversion time-consuming; hence, any volume reconstruction algorithm of practical interest should exhibit a computational complexity scaling (at most) linearly with the problem dimensions.

The first procedures proposed in the literature to reconstruct the volume of particles, namely the "Row-Action Methods", mainly focused on the complexity requirement. In this family of procedures, we acknowledge in particular the "Algebraic Reconstruction Technique" (ART) [25] and the "Multiplicative Algebraic Reconstruction Technique" (MART) [22], exploited in connection to TomoPIV in [34] and [19], respectively. While the latter class of algebraic procedures exploit one single pixel per iteration, their simultaneous counterparts - the "Simultaneous Iterative Reconstruction Technique" (SIRT) [13, 2] and "Simultaneous Multiplicative Algebraic Reconstruction Technique" (SMART) [10] - take benefit from the entire image at each step. SIRTs have been been advocated in [32] and [31], while SMART was exploited by Atkinson and Soria to decode the TomoPIV signal in [3].

The methodologies mentioned above suffer from a certain number of caveats. On the one hand, although the computational cost associated to each iteration is pretty low, their rates of convergence are usually quite slow [17] and highly depending on the choice of parameters [18]. As a consequence, while a few iterations of these procedures may lead to pretty poor reconstruction performance, letting them running up to convergence may lead to an unacceptable computational burden. On the second hand, the row-action methods do not fully exploit the knowledge available on the sought signal, namely a sparse distribution of small particles scattering a positive quantity of light energy. For example, (S)MARTs only take the nonnegativity of the solution into account whereas ARTs/SIRTs do neither exploit nonnegativity nor sparsity.

In order to address the latter issue, some authors have started tackling the TomoPIV problem by taking the sparsity constraint into account, see [6, 14, 7, 5, 36, 33, 21]. In [6], the authors have run extensive numerical assessment on greedy pursuit methods (e.g., "Orthogonal Matching Pursuit" (OMP) [30], "Subspace Pursuit" (SP) [15] and their bayesian counterparts [23]) applied to the TomoPIV volume reconstruction problem. In [14], Cornic et al explored the benefits of CoSaMP [27] to build a TomoPIV solution with a fixed number of non-zero coefficients. In $[7,5,36,33,21]$, sparse solutions of the volume reconstruction problem were searched by means of convex relaxation procedures. In particular, in [5] we proposed a general framework for the derivation of fast and tractable procedures exploiting both the nonnegativity and the sparsity of the solution. Our approach was based on the framework of (accelerated) proximal gradient procedures, a family of algorithms recently advocated in the community of machine 
learning to solve large-scale optimization problems, see e.g., [29].

In this paper, we target again the problem of devising fast and reliable (in particular, exploiting both the nonnegativity and the sparsity of the solution) volume reconstruction methods by using a different point of view. More specifically, we emphasize that the TomoPIV volume reconstruction task can be addressed by resorting to the powerful "Alternating Direction Method of Multipliers" (ADMM) [16]. Similarly to the proximal gradient method promoted in [5], ADMM is an optimization procedure which has become popular recently in the machine-learning community for solving large-scale optimization problems. In this paper, we show that the proposed procedure based on ADMM has some desirable features for the volume reconstruction problem. In particular, on top of accounting for both the nonnegativity and the sparsity of the solution, the proposed procedure enjoys the following properties: i) it allows for an explicit handling of the noise level affecting the observations; ii) it possesses - in certain variants of the proposed paradigm - the same complexity per iteration as the "RowAction Methods", but exhibits a faster speed of convergence than the latter.

The rest of the paper is organized as follows. In Section 2, we introduced the notational convention which will be used in the paper. Section 3 formalizes the 2D$3 \mathrm{D}$ projection model of the particles distribution and discloses a brief chronicle of other modeling choices throughout the years. We introduce, in Section 4, the ADMM framework and study its applicability to the TomoPIV task. Section 5 assesses the reconstruction quality of the proposed algorithm out of a synthetic data set. A discussion sums-up our contribution in Section 6.

\section{Notations}

All the vectors involved in our derivations are column vectors and are denoted by lowercase bold letters, as $\mathbf{x}$. Matrix quantities are denoted by uppercase bold letters, as $\mathbf{X}$. Sets are represented by calligraphic letters except for $\mathbb{R}$ and $\mathbb{R}_{+}$which respectively stand for the set of real numbers and the positive orthant. We define the characteristic function $\mathbb{I}_{\mathcal{X}}(\mathbf{x})$ of a set $\mathcal{X}$ as

$$
\mathbb{I}_{\mathcal{X}}(\mathbf{x})= \begin{cases}0 & \text { if } \mathbf{x} \in \mathcal{X} \\ +\infty & \text { otherwise }\end{cases}
$$

The projection operator of a point $\mathbf{v}$ onto a convex set $\mathcal{X}$ is defined as:

$$
\Pi_{\mathcal{X}}(\mathbf{v})=\underset{\mathbf{x} \in \mathcal{X}}{\arg \min }\|\mathbf{x}-\mathbf{v}\|_{2} .
$$

\section{The TomoPIV Reconstruction Problem}

A careful mathematical abstraction of the interaction between the particles' distribution and their projection on the images is of paramount importance for the accuracy of the subsequent volume estimation problem. The latter is inferred from the knowledge of the specifics of the experimental system. 


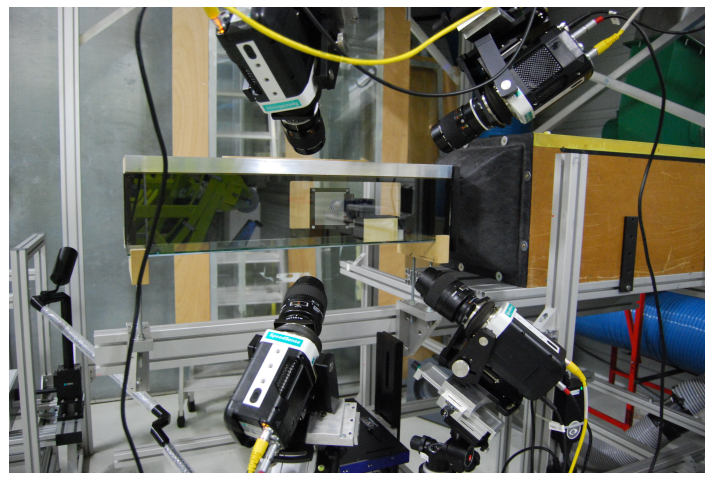

(a)

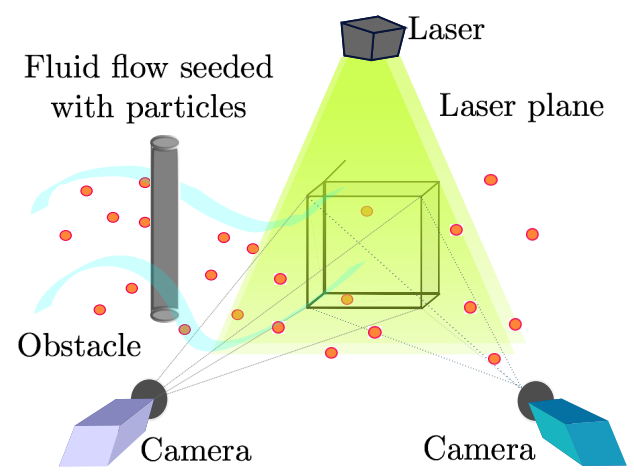

(b)

Figure 1: Left: an experimental 4-cameras (CLaVision TomoPIV setting located at the research center Irstea Rennes. Right: a 2-cameras schematic translation of the TomoPIV setting at a given time frame.

The TomoPIV technique aims at synchronously imaging lightly seeded particles at high update rates from a finite number of different views. Figure 1(a) captures an experimental setup, while Figure 1(b) faithfully illustrates its schematic counterpart. In a nutshell, a user-defined measurement volume is illuminated by a thick laser beam. Passive tracer particles are then immersed in the region of interest. The planes of the cameras placed around the illuminated region gather the light scattered by the tracers. One can thus define a projection model between the 3D and 2D spaces based upon the results of a calibration procedure and the physical knowledge of the process. Due to the numerous degrees of freedom when designing this so-called direct model, various recipes have been churned out to mirror the creation of the particles' images. Nevertheless, all the models proposed in the literature takes the same mathematical form:

$$
\mathbf{y}=\mathbf{A x}
$$

where $\mathbf{y} \in \mathbb{R}^{m}$ collects the pixels from all the cameras and $\mathbf{x} \in \mathbb{R}^{n}$ gathers the intensities of the particles at some particular positions of the volume. The exact nature and definition of matrix $\mathbf{A}$ depends on the considered model. We expose below different methodologies used in the literature to build matrix $\mathbf{A}$.

We note that our derivations in the next sections are based on a general model of the form of Equation (2). Therefore, all our results apply to the different models described hereafter.

\subsection{The Airy-spot Model}

The hardship of developing a realistic model of the TomoPIV image formation comes from the numerous physical parameters acting on the PIV image formation. For example, in a typical optical setup, the light intensity decreases throughout the volume in a way inversely proportional to the square of the distance from the light source. As a 
consequence, the particles' illumination is not uniform. More unknowns are due to the physical properties of the seeders. First, their small diameter (e.g., up to 10 microns for air experiments) influences the way they scatter the light; in fact, accurate PIV renditions are enabled by the ability of the particles to redirect the light received from the laser source in the direction of the camera. Moreover, based upon the ratio between the wavelength of the source light and the diameter of the particles, the quantity of scattered light can be appreciated by following the Mie scattering model [8]. Finally, due to intrinsic properties of the imaging lenses, the projection of a small particle onto an image impacts an aggregation of adjacent pixels; the latter then form an airy spot instead of a dot-like projection.

It is usually difficult to precisely account for all the physical phenomena described above. Hence, Champagnat et al [12] recently suggested a simplified physics-driven model describing the formation of the image airy-spots encountered in TomoPIV. More specifically, assuming there are $p$ particles in the region of interest (located at positions $\left\{\mathbf{k}_{i}\right\}_{i=1}^{p} \in \mathbb{R}^{3}$ in the volume), Champagnat et al proposed to model the image intensity $y$ at a point $\mathbf{u} \in \mathbb{R}^{2}$ of the image (of a given camera) as:

$$
y(\mathbf{u})=\sum_{i \in\{1, \ldots, p\}} A\left(\mathbf{u}-P\left(\mathbf{k}_{i}\right)\right) x_{i},
$$

where $P: \mathbb{R}^{3} \rightarrow \mathbb{R}^{2}$ is the projection operator of a $3 \mathrm{D}$ point in the volume onto the image plane and is highly depending on the optical setup of the imaging system, while the function $A: \mathbb{R}^{2} \rightarrow \mathbb{R}_{+}$is the so-called Point Spread Function (PSF) modeling the formation of the airy-spots on the image. As an example, Champagnat et al write $A(\cdot)$ as the convolution between a Gaussian and a gate function; the former accounting for blur and defocalisation while the latter describes the spatial integration over the detector's surface.

The model defined in Equation (3) can be repeated for all the cameras of the TomoPIV system; the definition of $P$ then changes as a function of the position of the camera. This model will be referred to as the "Airy-spot" model in the sequel. It is interesting to note that the particles are here assumed to be located at single (zero-dimensional) points $\left\{\mathbf{k}_{i}\right\}_{i=1}^{p}$. This is in contrast with the "Tomographic" model described in the next section, in which it is assumed that the particle are spread in the volume.

A discrete version of Equation (3) can be found by imposing to $\mathbf{u}$ and $\mathbf{k}_{i}$ to take on values in some discrete sets. More specifically, let $\left\{\mathbf{u}_{1}, \ldots, \mathbf{u}_{m}\right\}$ (resp. $\left.\left\{\mathbf{k}_{1}, \ldots, \mathbf{k}_{n}\right\}\right)$ denote the points of a 2D (resp. a 3D) cartesian grid covering the 2D images (resp. 3D volume). Then the linear model can be expressed as in Equation (2), where $\mathbf{y} \in \mathbb{R}^{m}$ collects the pixels from all the camera planes at positions $\left\{\mathbf{u}_{1}, \ldots, \mathbf{u}_{m}\right\}, \mathbf{x} \in \mathbb{R}^{n}$ gathers the intensities of the particles located at positions $\left\{\mathbf{k}_{1}, \ldots, \mathbf{k}_{n}\right\}$ in the volume, while the matrix $\mathbf{A} \in \mathbb{R}^{m} \times \mathbb{R}^{n}$ encodes the projection of the $3 \mathrm{D}$ space onto the images via Equation (3), that is $a_{i j}=A\left(\mathbf{u}_{i}-P\left(\mathbf{k}_{j}\right)\right)$. We mention that other developments in the literature account for the PSF in the image formation model in $[39,40,44]$. 


\subsection{The Tomographic Model}

The TomoPIV image formation model introduced in the seminal paper [19] is based on the following hypotheses: i) the particles' intensity is spatially distributed in the volume (a convention of a spreading-width of 3 voxels is typically adopted in the literature); ii) a pixel's intensity results from the integration of the particles' intensity along the line-of-sight passing thought the pixel.

It is interesting to underline the differences between the tomographic model described here and the model specified by Equation (3). The tomographic model assumes that the particles have some spatial volume; this in contrast with the model described in the previous section where dot-like particles were assumed. Moreover, in the tomographic model, the pixel intensity is the result of an integration process whereas in Equation (3), the pixel intensity is the consequence of an aperture diffraction. Although, the model described by Equation (3) seems to be in better adequation with the physical process underlying the image formation, the tomographic model has been used with success in many contributions of the TomoPIV literature. Papers treating the TomoPIV with great mathematical underpinning by Petra et al cast a similar blob model [35, 34, 37]. Among other, Worth and Nickels mention a comparable model in $[45]$.

Interestingly, the discretization of the tomographic model takes the same form as in Equation (2) but the computation of the corresponding encoding dictionary A, however, is very much different from the one in Equation (3). In fact, its calculation pattern is taken out of the classical X-Ray Tomography applications - for a full depiction of the process the author should refer to [11], from where the TomoPIV experimental scenario uproots its name.

\subsection{Promoted Choice}

We have reviewed, throughout this section, different abstractions to digitally mimic the analogical TomoPIV signal and its projection onto the image planes. In a nutshell, we distinguish mainly between the particle approach put forth by Champagnat et al in [12] (see Section 3.1) and the classical blob-oriented model introduced by Elsinga et al in [19] (see Section 3.2). While the former builds (and reconstructs) a sparse vector indicating the position of a particle within a voxel and its associated intensity, the latter obtains a PSF-like footprint on the 2D images by building the ground truth as 3D Gaussian blobs in the space (and subsequently reconstructing a blob vector as well). The particlebased approach models with high fidelity the physical mannerism of the projection of small-sized particles onto the image planes; the so-obtained, very sparse, estimation is however unsuited for the subsequent dense, correlation-based velocity estimation techniques classically used in the literature. The smoother blob-reconstruction, on the other hand, helps avoiding peak-locking effect and thus outputs reliable velocity fields. We propose, in this paper, to take benefit from both programs and set forth a particleblob hybrid approach written in the form of Equation (2). The latter seeks the 3D 
density signal in a (very) sparse space, but enables the blunt construction of a dense space appropriate for the velocity search space. For doing so, we rely on a discrete approximation of the PSF, where the encoding matrix $\mathbf{A}$ has been computed according to Appendix A.

\section{TomoPIV meets ADMM}

In this section, we expose our methodology based on ADMM to solve the volume reconstruction task. We start by expressing the TomoPIV problem from an optimization viewpoint in Section 4.1. We then depict the general ADMM framework in Section 4.2 and particularize the latter to the TomoPIV problem in Section 4.3. Finally, we discuss the complexity of the resulting algorithm and propose a low-complexity variant in Section 4.4.

\subsection{TomoPIV Reconstruction as a Convex Optimization Problem}

The derivation of our reconstruction procedure is driven by the two following observations. First, due to the limited number of observations available, the TomoPIV system depicted by Equation (2) has generally infinitely many solutions. A common modus operandi when dealing with signal reconstruction from limited data is to resort to some known information on the original signal in order to single out a proper solution. For doing so in our application, we wish to capitalize on the known features of the TomoPIV signal, i.e., its non-negativity and its sparsity. Second, the data collected by the cameras are often corrupted by different types of noise (for example acquisition or modeling noises). Hence, the sought particles' density $\mathbf{x}$ and the collected observations y rarely perfectly match model (2). In practice, one should therefore allow our volume estimate, say $\mathbf{x}^{\star}$, to suffer from some (limited) discrepancies with respect to the considered model.

In order to take these two remarks into account, we consider the following optimization problem:

$$
\mathbf{x}^{\star}=\underset{\mathbf{x}}{\arg \min } \mathrm{r}(\mathbf{x}) \text { such that }\|\mathbf{y}-\mathbf{A x}\|_{2} \leq \varepsilon,
$$

where $\varepsilon \geq 0$ and $\mathrm{r}: \mathbb{R}^{n} \rightarrow \mathbb{R}_{+}$is some "regularization" function. In this problem, the presence of noise on the data is accounted for via the constraint " $\|\mathbf{y}-\mathbf{A x}\|_{2} \leq \varepsilon$ " (in fact, deviations from the noiseless observation model (2) are allowed as soon as $\varepsilon>0$ ). Moreover, depending on the choice of $r$, one may enforce the solution of (4) to exhibit some particular features. In particular, we discuss hereafter different choices of $r$ which may fulfill our sparsity and non-negativity requirements.

An ideal choice for $\mathrm{r}$ to promote the sparsity of $\mathrm{x}^{\star}$ is given by the (so-called) " $\ell_{0}$ norm", i.e., $\mathrm{r}(\mathbf{x})=\|\mathbf{x}\|_{0} \doteq \sum_{i=1}^{n}\left|x_{i}\right|^{0}$, which counts the number of nonzero elements in its argument. Unfortunately, the $\ell_{0}$ norm is a non-smooth, non-convex function and 
setting $\mathrm{r}(\mathbf{x})=\|\mathbf{x}\|_{0}$ in (4) generally results in an intractable problem, see [20, Section 2.3]. Hence, in practice, the $\ell_{1}$ norm:

$$
\mathrm{r}(\mathrm{x})=\|\mathbf{x}\|_{1} \doteq \sum_{i=1}^{n}\left|x_{i}\right|
$$

is often preferred as a convex surrogate to the $\ell_{0}$ norm. Interestingly, it has been shown in many theoretical works (see e.g., [20, Chapter 4]) that considering the $\ell_{0}$ or the $\ell_{1}$ norms in the problem defined in (4) essentially leads to the same results, as long as the ground-truth vector from which the observations originate is sparse enough. In the context of TomoPIV, since the true particles' distribution is typically sparse, using $\mathrm{r}(\mathbf{x})=\|\mathbf{x}\|_{1}$ in (4) is likely to lead to a sparse solution $\mathbf{x}^{\star}$.

The non-negativity constraints can be implicitly enforced by considering the regularization function as the indicator function of the positive orthant, i.e.,

$$
\mathrm{r}(\mathbf{x})=\mathbb{I}_{\mathbb{R}_{+}^{n}}(\mathbf{x}) .
$$

Finally, taking (5) and (6) into account, it is easy to see that one can enforce both the non-negativity and the sparsity of the solution by simply concatenating the regularization terms defined above, that is :

$$
\mathrm{r}(\mathbf{x})=\|\mathbf{x}\|_{1}+\mathbb{I}_{\mathbb{R}_{+}^{n}}(\mathbf{x}) .
$$

Let us note that the different regularization functions defined in (5), (6) and (7) are all convex. Since the constraint " $\|\mathbf{y}-\mathbf{A x}\|_{2} \leq \varepsilon$ " is also convex, problem (4) is convex irrespective of the choice of the cost function (5)-(7) and can therefore be solved

efficiently via state-of-the-art optimization techniques. This is the purpose of the rest of this section. In the next section, we describe the general update rules of the ADMM procedure; then, in the two subsequent sections, we particularize the latter to problem $(4)$.

\subsection{Quick Tutorial on ADMM}

ADMM focusses on the following type of optimization problems

$$
\min _{\mathbf{t}, \mathbf{z}} \mathrm{f}(\mathbf{t})+\mathrm{g}(\mathbf{z}) \text { such that } \mathbf{D t}+\mathbf{z}=0
$$

where $\mathrm{f}: \mathbb{R}^{a} \rightarrow \mathbb{R}, \mathrm{g}: \mathbb{R}^{b} \rightarrow \mathbb{R}$ are closed, proper and convex functions. The ADMM is an iterative procedure searching for a minimizer of problem (8) via the following recursion:

$$
\begin{aligned}
& \mathbf{t}^{(k+1)}=\underset{\mathbf{t}}{\arg \min }\left\{\mathrm{f}(\mathbf{t})+\frac{\rho}{2}\left\|\mathbf{D t}+\mathbf{z}^{(k)}+\mathbf{u}^{(k)}\right\|_{2}^{2}\right\} \\
& \mathbf{z}^{(k+1)}=\underset{\mathbf{z}}{\arg \min }\left\{\mathrm{g}(\mathbf{z})+\frac{\rho}{2}\left\|\mathbf{D} \mathbf{t}^{(k+1)}+\mathbf{z}+\mathbf{u}^{(k)}\right\|_{2}^{2}\right\} \\
& \mathbf{u}^{(k+1)}=\mathbf{u}^{(k)}+\mathbf{D t}^{(k+1)}+\mathbf{z}^{(k+1)},
\end{aligned}
$$


where $\rho>0$ is some arbitrary parameter and $(k)$ is the index of the iteration. We refer the reader to the very good tutorial on ADMM [9] for an explanation of the rationale behind this type of methodology.

ADMM has recently caught the attention of the signal-processing community for several reasons. First, the ADMM recursion defined in (8)-(10) converges to a solution of problem (8) under very general conditions, see [9, section 3.2]. In particular, the conditions on $\mathrm{f}$ and $\mathrm{g}$ are very mild: they are not required to be differentiable and can take on infinite values. Therefore, the problem stated in Equation (8) encompasses a large number of optimization problems as particular cases. Second, the algorithmic scheme defined in (8)-(10) has been shown empirically to converge to modest accuracy to a solution of (8) in only a few tens of iterations. Finally, the optimization problems involved in the updates of $\mathbf{t}^{(k+1)}$ and $\mathbf{z}^{(k+1)}$ admit very fast solutions or closed-form expressions in many setups. These two last features make ADMM very appealing in large-scale problems where modest accuracy is often sufficient but complexity load is of utmost importance.

\subsection{ADMM Applied to the TomoPIV Problem}

In this section, we particularize the generic ADMM recursions stated in (8)-(10) to the TomoPIV reconstruction problem (4). Our ensuing developments are inspired from the "C-SALSA" algorithm introduced in [1]. In order to take the algorithmic cue from the upper-evoked scheme, we can express the problem stated in Equation (4) as follows:

$$
\min _{\mathbf{x}} r(\mathbf{x})+\mathbb{I}_{\mathcal{B}(\mathbf{y}, \varepsilon)}(\mathbf{A x})
$$

where $\mathcal{B}(\mathbf{y}, \varepsilon)=\left\{\mathbf{v} \in \mathbb{R}^{m}:\|\mathbf{y}-\mathbf{v}\|_{2} \leq \varepsilon\right\}$ is the $\ell_{2}$ ball of radius $\varepsilon$ centered on $\mathbf{y}$. In fact, the constraint " $\|\mathbf{y}-\mathbf{A x}\|_{2} \leq \varepsilon$ " pitched in problem (4) and accounting for the presence of noise on the observations is implicitly injected in problem (11) as the second term of the cost function, that is the indicator function of the $\ell_{2}$ ball. Then, letting $\mathbf{z}=\left[\begin{array}{ll}\mathbf{z}_{1}^{T} & \mathbf{z}_{2}^{T}\end{array}\right]^{T} \in \mathbb{R}^{m+n}$, we can easily re-express (11) in the same form as problem $(8)$ :

$$
\min _{\mathbf{x}, \mathbf{z}_{1}, \mathbf{z}_{2}} \mathrm{r}\left(\mathbf{z}_{1}\right)+\mathbb{I}_{\mathcal{B}(\mathbf{y}, \varepsilon)}\left(\mathbf{z}_{2}\right) \text { such that }\left[\begin{array}{c}
\mathbf{I}_{n} \\
\mathbf{A}
\end{array}\right] \mathbf{x}-\left[\begin{array}{l}
\mathbf{z}_{1} \\
\mathbf{z}_{2}
\end{array}\right]=0,
$$

where (12) matches with (8) by making the following substitutions:

$$
\begin{aligned}
& \mathbf{t}=\mathbf{x}, \\
& \mathrm{f}(\mathbf{x})=1, \\
& \mathrm{~g}(\mathbf{z})=\mathrm{r}\left(\mathbf{z}_{1}\right)+\mathbb{I}_{\mathcal{B}(\mathbf{y}, \varepsilon)}\left(\mathbf{z}_{2}\right), \\
& \mathbf{D}=-\left[\begin{array}{c}
\mathbf{I}_{n} \\
\mathbf{A}
\end{array}\right] .
\end{aligned}
$$

Particularizing the ADMM update rules defined in (8)-(10) to the targeted problem (12), we then obtain the following recursions: 


$$
\begin{aligned}
& \mathbf{x}^{(k+1)}=\underset{\mathbf{x}}{\arg \min }\left\{\left\|\mathbf{x}-\mathbf{z}_{1}^{(k)}-\mathbf{u}_{1}^{(k)}\right\|_{2}^{2}+\frac{\rho}{2}\left\|\mathbf{A} \mathbf{x}-\mathbf{z}_{2}^{(k)}-\mathbf{u}_{2}^{(k)}\right\|_{2}^{2}\right\} \\
& \left\{\begin{array}{l}
\mathbf{z}_{1}^{(k+1)}=\underset{\mathbf{z}_{1}}{\arg \min }\left\{\rho^{-1} \mathrm{r}\left(\mathbf{z}_{1}\right)+\frac{1}{2}\left\|\mathbf{z}_{1}+\mathbf{u}_{1}^{(k)}-\mathbf{x}^{(k+1)}\right\|_{2}^{2}\right\} \\
\mathbf{z}_{2}^{(k+1)}=\underset{\mathbf{z}_{2}}{\arg \min }\left\{\mathbb{I}_{\mathcal{B}(\mathbf{y}, \varepsilon)}\left(\mathbf{z}_{2}\right)+\frac{1}{2}\left\|\mathbf{z}_{2}+\mathbf{u}_{2}^{(k)}-\mathbf{A} \mathbf{x}^{(k+1)}\right\|_{2}^{2}\right\}
\end{array}\right. \\
& \left\{\begin{array}{l}
\mathbf{u}_{1}^{(k+1)}=\mathbf{u}_{1}^{(k)}-\mathbf{x}^{(k+1)}+\mathbf{z}_{1}^{(k+1)} \\
\mathbf{u}_{2}^{(k+1)}=\mathbf{u}_{2}^{(k)}-\mathbf{A} \mathbf{x}^{(k+1)}+\mathbf{z}_{2}^{(k+1)} .
\end{array}\right.
\end{aligned}
$$

The minimization problems defined in (14)-(15) admit simple analytical solutions. First, the $\mathbf{x}$-update (14) is tantamount to solving a least-square problem, whose closedform solution is given by

$$
\mathbf{x}^{(k+1)}=\left(\mathbf{I}_{n}+\frac{\rho}{2} \mathbf{A}^{T} \mathbf{A}\right)^{-1}\left(\mathbf{z}_{1}^{(k)}+\mathbf{u}_{1}^{(k)}+\frac{\rho}{2} \mathbf{A}^{T}\left(\mathbf{z}_{2}^{(k)}+\mathbf{u}_{2}^{(k)}\right)\right) .
$$

Second, the solution of the $\mathbf{z}_{1}$-minimization problem in (15) is given by the "proximal" function $\ddagger$ of $\rho^{-1} \mathrm{r}(\mathbf{x})$, denoted hereafter as $\operatorname{prox}_{\rho^{-1} \mathrm{r}}(\cdot)$, that is:

$$
\mathbf{z}_{1}^{(k+1)}=\operatorname{prox}_{\rho^{-1} \mathrm{r}}\left(\mathbf{x}^{(k+1)}-\mathbf{u}_{1}^{(k)}\right) .
$$

As long as the particular regularization functions $\mathrm{r}(\mathbf{x})$ defined in (5)-(7) are concerned, the proximal operator $\operatorname{prox}_{\rho^{-1} \mathrm{r}}(\cdot)$ takes very simple analytical forms. For example, when $\mathrm{r}(\mathbf{x})=\|\mathbf{x}\|_{1}$, the $i$ th component of the output of the proximal operator evaluated at some vector $\mathbf{v} \in \mathbb{R}^{n}$ can be written as

$$
\left(\operatorname{prox}_{\rho^{-1}\|\cdot\|_{1}}(\mathbf{v})\right)_{i}= \begin{cases}v_{i}-\rho^{-1} & v_{i} \geq \rho^{-1} \\ 0 & \left|v_{i}\right| \leq \rho^{-1} \\ v_{i}+\rho^{-1} & v_{i} \leq-\rho^{-1}\end{cases}
$$

This operator is often referred to as "soft thresholding" because it zeroes all the components of the input $\mathbf{v}$ whose amplitude is below $\rho^{-1}$ and slightly decreases the amplitude of the other coefficients. Similarly, when $r(\mathbf{x})=\mathbb{I}_{\mathbb{R}_{+}^{n}}(\mathbf{x})$, the $i$ th output of the proximal operator applied to some input vector $\mathbf{v} \in \mathbb{R}^{n}$ can simply be expressed as

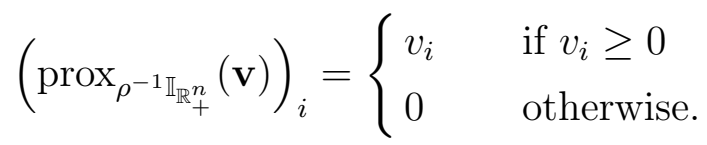

Finally, the output of the proximal operator corresponding to $r(\mathbf{x})=\lambda\|\mathbf{x}\|_{1}+\mathbb{I}_{\mathbb{R}_{+}^{n}}(\mathbf{x})$ reads as

$$
\left(\operatorname{prox}_{\rho^{-1}\left(\|\cdot\|_{1}+\mathbb{I}_{+}^{n}\right)}(\mathbf{v})\right)_{i}= \begin{cases}v_{i}-\rho^{-1} & v_{i} \geq \rho^{-1} \\ 0 & \text { otherwise. }\end{cases}
$$

$\ddagger$ The reader is invited to consult the tutorial paper [29] for a good introduction to the theory of proximal operators. 
We refer the reader to [29, Chapter 6] for a detailled derivation of these analytical expressions.

Regarding the update of $\mathbf{z}_{2}$, we may notice that the minimization problem in (15) can also be rewritten as

$$
\mathbf{z}_{2}^{(k+1)}=\underset{\mathbf{z}_{2} \in \mathcal{B}(\mathbf{y}, \varepsilon)}{\arg \min }\left\|\mathbf{z}_{2}+\mathbf{u}_{2}^{(k)}-\mathbf{A} \mathbf{x}^{(k+1)}\right\|_{2}^{2}=\Pi_{\mathcal{B}(\mathbf{y}, \varepsilon)}\left(\mathbf{A} \mathbf{x}^{(k+1)}-\mathbf{u}_{2}^{(k)}\right),
$$

that is, corresponds to the projection of $\mathbf{A} \mathbf{x}^{(k+1)}-\mathbf{u}_{2}^{(k)}$ onto the $\ell_{2}$ ball $\mathcal{B}(\mathbf{y}, \varepsilon)$. As shown in [1, Section 3], this kind of operation admits a simple analytical expression. More specifically, the projector of some vector $\mathbf{v} \in \mathbb{R}^{m}$ onto $\mathcal{B}(\mathbf{y}, \varepsilon)$ can be written as

$$
\Pi_{\mathcal{B}(\mathbf{y}, \varepsilon)}(\mathbf{v})=\mathbf{y}+\left\{\begin{array}{cl}
\varepsilon \frac{\mathbf{v}-\mathbf{y}}{\|\mathbf{v}-\mathbf{y}\|_{2}} & \text { if }\|\mathbf{v}-\mathbf{y}\|_{2}>\varepsilon \\
\mathbf{v}-\mathbf{y} & \text { if }\|\mathbf{v}-\mathbf{y}\|_{2} \leq \varepsilon
\end{array}\right.
$$

Table 1 sums-up the updates implied in solving the problem (11) with $\mathrm{r}(\mathbf{x})=$

\begin{tabular}{|c|c|c|}
\hline Update & Equation number & Solution \\
\hline $\mathbf{x}^{(k+1)}$ & $(17)$ & $\left(\mathbf{I}_{n}+\frac{\rho}{2} \mathbf{A}^{T} \mathbf{A}\right)^{-1}\left(\mathbf{z}_{1}^{(k)}+\mathbf{u}_{1}^{(k)}+\frac{\rho}{2} \mathbf{A}^{T}\left(\mathbf{z}_{2}^{(k)}+\mathbf{u}_{2}^{(k)}\right)\right)$ \\
\hline $\mathbf{z}_{1}^{(k+1)}$ & $(21)$ & $\begin{cases}\left(\mathbf{A} \mathbf{x}^{(k+1)}-\mathbf{u}_{2}^{(k)}\right)_{i}-\rho^{-1} & \text { if }\left(\mathbf{A} \mathbf{x}^{(k+1)}-\mathbf{u}_{2}^{(k)}\right)_{i} \geq \rho^{-1} \\
0 & \text { otherwise }\end{cases}$ \\
\hline $\mathbf{z}_{2}^{(k+1)}$ & $(23)$ & $\mathbf{y}+\left\{\begin{array}{cl}\varepsilon \frac{\mathbf{A} \mathbf{x}^{(k+1)}-\mathbf{u}_{2}^{(k)}-\mathbf{y}}{\left\|\mathbf{A} \mathbf{x}^{(k+1)}-\mathbf{u}_{2}^{(k)}-\mathbf{y}\right\|_{2}} & \text { if }\left\|\mathbf{A} \mathbf{x}^{(k+1)}-\mathbf{u}_{2}^{(k)}-\mathbf{y}\right\|_{2}>\varepsilon \\
\mathbf{A} \mathbf{x}^{(k+1)}-\mathbf{u}_{2}^{(k)}-\mathbf{y} & \text { if }\left\|\mathbf{A} \mathbf{x}^{(k+1)}-\mathbf{u}_{2}^{(k)}-\mathbf{y}\right\|_{2} \leq \varepsilon\end{array}\right.$ \\
\hline $\mathbf{u}_{1}^{(k+1)}$ & $(16)$ & $\mathbf{u}_{1}^{(k)}-\mathbf{x}^{(k+1)}+\mathbf{z}_{1}^{(k+1)}$ \\
\hline $\mathbf{u}_{2}^{(k+1)}$ & $(16)$ & $\mathbf{u}_{2}^{(k)}-\mathbf{A} \mathbf{x}^{(k+1)}+\mathbf{z}_{2}^{(k+1)}$ \\
\hline
\end{tabular}
$\|\mathbf{x}\|_{1}+\mathbb{I}_{\mathbb{R}_{+}^{n}}(\mathbf{x})$, i.e., Equations (14)-(16) and their respective solutions.

Table 1: Recap of the ADMM updates involved in solving problem (11) with $\mathrm{r}(\mathbf{x})=$ $\|\mathbf{x}\|_{1}+\mathbb{I}_{\mathbb{R}_{+}^{n}}(\mathbf{x})$.

\subsection{Complexity}

In this section, we discuss the complexity of the ADMM procedure described in Table 1 and propose a variant which improves its computational complexity.

The complexity of the ADMM procedure described above is dominated by the $\mathbf{x}$ update (17). Indeed, let us first notice that the matrix $\mathbf{A} \in \mathbb{R}^{m \times n}$ appearing in our model (2) is typically very sparse, so that the multiplication of this matrix by a vector has generally a complexity of $\mathcal{O}(n)$. Next, as emphasized in the previous section, the update of $\mathbf{z}^{(k)}$ only requires very simple thresholding operations, see (19)-(23). The complexity associated to this step thus scales as $\mathcal{O}(m+n)$. Similarly, the update of $\mathbf{u}^{(k)}$ only requires vector summation and has therefore a complexity evolving as $\mathcal{O}(n)$. The $\mathbf{x}$-update (17) is more critical since it involves the inversion of the matrix $\left(\mathbf{I}_{n}+\frac{\rho}{2} \mathbf{A}^{T} \mathbf{A}\right) \in \mathbb{R}^{n \times n}$. A brute-force inversion of this matrix requires a complexity scaling as $\mathcal{O}\left(n^{3}\right)$ which is 
clearly prohibitive in the context of TomoPIV. In the rest of this section, we emphasize that an exact inversion of $\left(\mathbf{I}_{n}+\frac{\rho}{2} \mathbf{A}^{T} \mathbf{A}\right)$ can be carried out with a complexity scaling as $\mathcal{O}\left(m^{2} n\right)$ by taking its particular structure into account. We then suggest an approximate update of $\mathbf{x}^{(k)}$ which leads to an overall complexity of $\mathcal{O}(n)$.

First, note that the inverse of $\left(\mathbf{I}_{n}+\frac{\rho}{2} \mathbf{A}^{T} \mathbf{A}\right)$ can be alternatively expressed as

$$
\left(\mathbf{I}_{n}+\frac{\rho}{2} \mathbf{A}^{T} \mathbf{A}\right)^{-1}=\mathbf{I}_{n}-\mathbf{A}^{T}\left(\frac{2}{\rho} \mathbf{I}_{m}+\mathbf{A} \mathbf{A}^{T}\right)^{-1} \mathbf{A}
$$

which only requires the inversion of a $m \times m$ matrix $\left(\frac{2}{\rho} \mathbf{I}_{m}+\mathbf{A A}^{T}\right)$. Moreover, the matrix to invert does not vary from iteration to iteration; one can therefore take benefit from a Cholesky factorization [38] of $\frac{2}{\rho} \mathbf{I}_{m}+\mathbf{A A}^{T}$ to evaluate Equation (17) with a complexity scaling as $\mathcal{O}\left(m^{2} n\right)$, see [29, Section 6.1.1]. This approach requires however the storage of the (dense) $m \times m$ matrix of the Cholesky decomposition.

Instead of using the analytical solution (17), another route to solve the least-square problem stated in (14) consists in resorting to iterative search techniques such as, for example, the "Conjugate-Gradient Method" (CGM). The CGM algorithm is broadly employed to solve large systems of linear equations; for an intuitive, easy to understand tutorial, the reader can refer to [41]. In a nutshell, the CGM is a descent method in which the descent direction used at each iteration is "conjugated" to the directions exploited during the previous iterations. For the purpose of our discussion, the key elements to notice are: $i$ ) the complexity of the CGM is dominated by the evaluation of these descent directions; ii) the latter can be evaluated easily from the knowledge of the gradient of the function to minimize. Now, as far as the resolution of problem (14) is concerned, the gradient of the cost function takes the following simple form:

$$
\left(\mathbf{I}_{n}+\frac{\rho}{2} \mathbf{A}^{T} \mathbf{A}\right) \mathbf{x}-\left(\mathbf{z}_{1}^{(k)}+\mathbf{u}_{1}^{(k)}+\frac{\rho}{2} \mathbf{A}^{T}\left(\mathbf{z}_{2}^{(k)}+\mathbf{u}_{2}^{(k)}\right)\right) .
$$

In particular, the evaluation of the above expression only requires matrix-vector multiplications. Each iteration of the CGM applied to (14) has therefore a complexity scaling as $\mathcal{O}(n)$. Finally, let us emphasize that the convergence of the ADMM procedure (14)-(16) is still ensured if the solutions of the minimization problems (14)-(15) are computed with some finite precision, see [16]. In practice, a few iterations of CGM applied to (14) are thus usually sufficient to achieve such a level of precision. Hence, the overall complexity of the ADMM procedure based on CGM is of the order of $\mathcal{O}(n)$.

\section{Numerical Validation}

We will assess the reconstruction quality of a TomoPIV signal out of a synthetic data set. We will first lay out the specifics of the studied scenario. Then, we will examine the relative performance of the proposed algorithm with respect to the classical SMART ([10]) and its accelerated counterpart A-SMART ([5]). Finally, we will see how constraining ADMM to account for the sparsity of the signal influences the quality of the reconstruction. 


\subsection{Setup}

We consider an ill-posed problem inspired by the real-world application. First, we cast an area of interest in the illuminated seeded volume; the latter is seeded with particles placed at random positions. For straightforwardness, we make the assumption of a uniform illumination and we disregard the Mie scattering effect. Then, we digitize the volume into a $3 \mathrm{D}$ cartesian grid of $61 \times 61 \times 21$ voxels, with voxel unit set at 1 arbitrary units (arb. u.); this initial grid will later suit the subsequent velocity estimation - for an example, see [4, Section 4.4]. The origin of the scene frame is chosen so that it corresponds to the center of the 3D grid. Second, we place 4 cameras around the cuboid so that the latter projects entirely into each camera plane; each camera has a CCD sensor of $10 \times 10 \mathrm{arb}$. u. for a $61 \times 61$ resolution and a 4.4 focal distance. The sensors are designed according to a pin-hole model. Finally, given this setup and a perfectly known calibration, the images are synthesized according to Equation (2), where $y_{i}$ is affected by a Gaussian noise of zero mean and standard deviation equal to $0.1 y_{i}$. The volumetric signal is sought in agreement with our hybrid model defined by Equation (A.5); this involves the construction of a refined grid made up of $122 \times 122 \times 42$ sub-voxels. Then, we assume that the unknown positions of the center of the particles correspond to the centers of the given sub-voxels. The encoding dictionary $\mathbf{A} \in \mathbb{R}^{6724 \times 625128}$ translates into an $\frac{m}{n}=0.0108$ ratio between the number of observations and the number of unknowns and corresponds to a highly underdetermined problem. As a comparison, ratios $\frac{m}{n}=0.2105$ and $\frac{m}{n}=0.0141$ were considered in [33] and [14] respectively.

\subsection{Model and Metrics}

The widely-adopted metric to assess the quality of the reconstructed TomoPIV volume computes the normalized correlation between the ground truth and its estimated analogue: the so-called "Q criterion". The latter criterion is however not adapted to sparse particle-based reconstructions as the latter contain punctual voxelsized probes. To ensure the homogeneity with the community, we apply thus the Qcriterion to the blob-enhanced vector described by Equation (A.4). Let $\tilde{\mathbf{x}}$ be the ground truth distribution of particles in the volume. The blob density vector $\tilde{\mathbf{w}}$ can be deduced out of its particle-based counterpart $\tilde{\mathbf{x}}$ as $\tilde{\mathbf{w}}=\mathbf{G} \tilde{\mathbf{x}}$, where the matrix $\mathbf{G}$ takes on its columns the coefficients of the Gaussian functions centered on particles in the cuboid. We proceed in an analogue manner to build vector $\mathbf{w}^{\star}$ from the estimated vector $\mathbf{x}^{\star}$ that solves an optimization problem (4). The Q-criterion thus writes:

$$
\mathrm{Q}=\frac{\tilde{\mathbf{w}}^{T}}{\|\tilde{\mathbf{w}}\|_{2}} \frac{\mathbf{w}^{\star}}{\left\|\mathbf{w}^{\star}\right\|_{2}} .
$$

We evaluate the Q-criterion of the reconstruction with respect to the particles per

pixel (ppp) metric; the latter is computed as the ratio between the sparsity of the blob vector $\tilde{\mathbf{w}}$ and the number of pixels on one image. 


\subsection{ADMM vs. State-of-the-Art}

The complexity of the algorithms employed to solve problem (4) depends on the dimensions of the latter. Therefore, we resort to a pruning technique to reduce its magnitude. This can be done by exploiting the sparsity of the vectors $\mathbf{y}$ and $\mathbf{A}$ to determine which coefficients in the sought vector are likely to be non-zeros. The Feasible Reduced Set technique was introduced by Petra et al in the TomoPIV context [35]; the latter builds, as its name suggests, a reduced feasible set equivalent to the original one under minor constraints. For each experiment and for each ppp level, we eliminate the columns of the $\mathbf{A}$ matrix that do not contribute to the formation of the image. All of the results presented here-below - (A-)SMARTs, ADMMs - are computed on such pruned systems.

We run comparative numerical assessment of our newly advanced methods against state-of-the-art SMART [10] and its accelerated counterpart - that we have coined ASMART - introduced in our companion paper [5]. The variant of ADMM based on an iterative procedure (see Section 4.4) will be referred to as ADMM-CGM.

Since both SMART and A-SMART implicitly enforce the positivity of the sought solution, we first address the ADMM reconstruction with a positivity constraint. To do so, both the ADMM and the ADMM-CGM schemes will solve problem (11) with $\mathrm{r}(\mathbf{x})=\mathbb{I}_{\mathbb{R}_{+}^{n}}(\mathbf{x})$ (see Equation (6)). These derived forms will be alluded to as ADMM+ and ADMM-CGM+, respectively. We average our results on 30 experiments for each ppp value; the latter runs up until $\mathrm{ppp}=0.1$, which translates a sparsity of the blob vector running from roughly $4 \times 10^{3}$ to $10^{4}$ nonzero coefficients.

Figure 2 depicts the Q-criterion for the 4 selected algorithms at different moments of the convergence towards a solution; on the left, the Q-criterion is computed at $k=15$ iterations, while on the right the latter is recalculated for the solution obtained after $k=30$ iterations. Altogether, we notice that state-of-the-art SMART is outperformed by the newly generalized A-SMART and by the here-introduced ADMM+ procedure and its approximated variant ADMM-CGM+. The latter three, however, are serious competitors for the most accurate solution. On the graph on the left, we notice a clear superiority of the solutions output by ADMM+ and ADMM-CGM+ after 15 iterations, despite the faster decay towards convergence of A-SMART. On the right, the latter outperforms the ADMM-CGM+ for higher ppp values and almost levels with the ADMM+ procedure.

Naturally, the rates of convergence play a pivotal role on the reconstruction quality. ADMM and SMART are known to converge linearly (see [24] and [10], respectively) while the acceleration of SMART-like algorithms leads to a sublinear decay [28]. Figure 3 assesses the performance of the algorithms from a computational viewpoint. On the left, comparative graphs of the running time are depicted. On the right, Figure 3 takes a look at the convergence behavior for a median value of ppp, i.e., at 0.05; it depicts, in fact, the convergence slopes for each algorithms. We can rediscover the statements made here-above: after 15 iterations, the ADMMs are close together, with a clear superiority 
over the SMART and A-SMART, while after 30 iterations A-SMART continues its decay, while the ADMMs stagnate. What's more, the ADMMs decay might be quite lagging, but it reaches a satisfying solution within 10 iterations. Moreover, the solution output by the ADMMs after 10 iterations is closer to the ground truth than the one given by SMART after 30 iterations and than the one given by A-SMART after 20 iterations. This confirms that, while ADMM can be slow to converge with high accuracy, it provides a favorable solution in only a few iterations.

Q

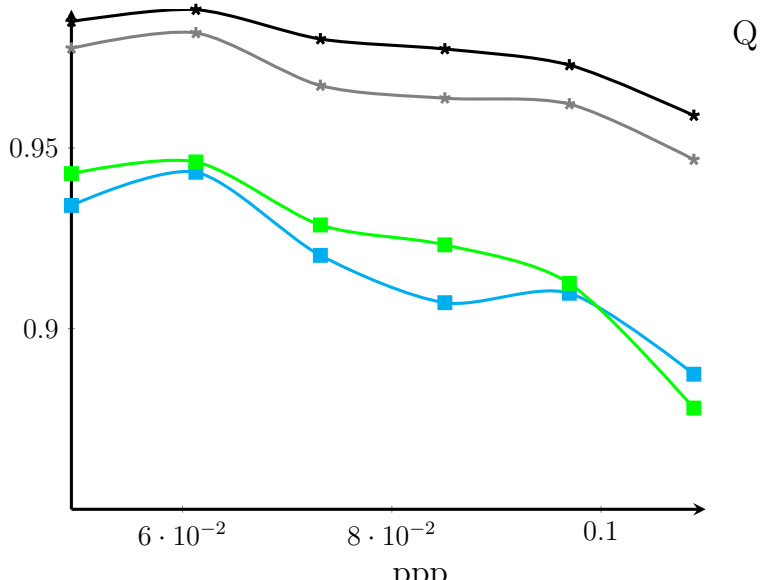

$\mathrm{Q}$

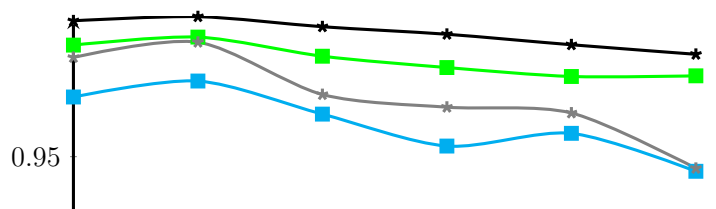

.9

$6 \cdot 10^{-2}$

ppp

$\rightarrow-$ SMART - A-SMART
- ADMM $+*$ ADMM-CGM +

Figure 2: Numerical assessment of the reconstruction quality of $\mathbf{w}^{\star}$ in terms of the normalized correlation against the "particles per pixel (ppp)" ratio. Left: The estimated blob-densities $\mathbf{w}^{\star}$ after $\mathbf{1 5}$ iterations. Right: The estimated blob-densities $\mathbf{w}^{\star}$ after $\mathbf{3 0}$ iterations.

\subsection{Constraining Sparsity with ADMM}

As mentioned before, one of the features of the TomoPIV signal is that is contains much more empty space than particles. This means that we are looking, in general, for a sparse solution to our problem. We propose to observe, in the same synthetic scenario, what are the advantages of constraining the sparsity of the solution. To do so, both the ADMM and the ADMM-CGM schemes will solve problem (11) with $\mathrm{r}(\mathbf{x})=\|\mathbf{x}\|_{1}+\mathbb{I}_{\mathbb{R}_{+}^{n}}(\mathbf{x})$ (see Equation (7)) and thus accounting for both the non-negativity and the sparsity of the signal. These derived forms will be denoted by ADMM $\ell_{1}+$ and $\mathrm{ADMM}-\mathrm{CGM} \ell_{1}+$, respectively. Figure 4 summarizes our assessment. As expected, the constrained counterparts of ADMM+ lead to a small increase in the quality of the reconstruction - particularly for low ppp values - as visible on the right of Figure 4. This is in part explained by the fact that the support vector (i.e., the vector corresponding to the non-zero coefficients) of the estimated solution is sparse. In fact, as the ppp increases, the ADMM+ and ADMM-CGM+ solutions become denser; this translates into myriads of spurious atoms of very low energy that affect the reconstruction and 

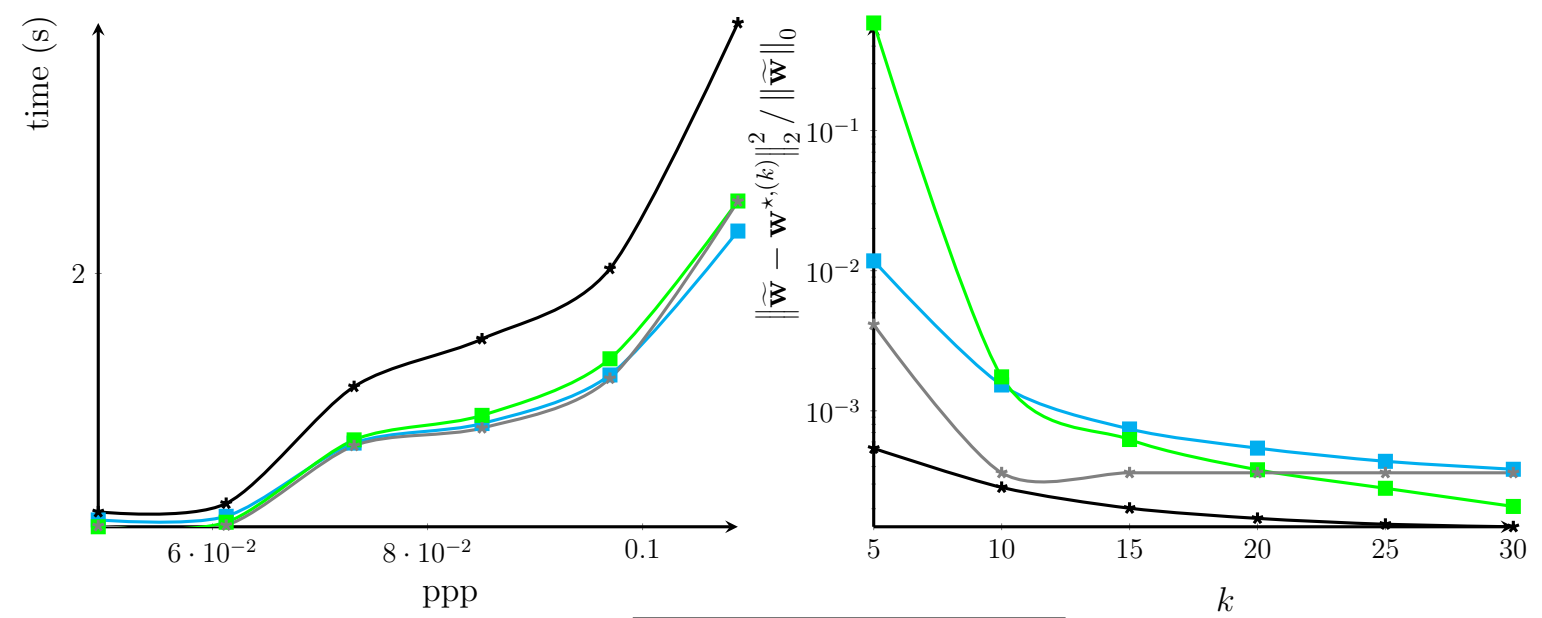

- SMART $-\frac{1}{-}$ A-SMART

*-ADMM+ *-ADMM-CGM+

Figure 3: Convergence analysis of the assessed algorithms. Left: Running time for 30 iterations against the ppp ratio. Right: Normalized distance of the solution at the $k$ th iteration $\mathbf{w}^{\star,(k)}$ to the ground truth value with respect to the number of iterations for $\mathrm{ppp}=0.05$.

thus, the Q criterion. However, the main advantage of constraining the solution with a $\ell_{1}$ norm has a positive impact on a the running time - as visible on the left of Figure 4 in particular for the ADMM paradigm. In fact, dealing with sparse vectors alleviates the complexity of the algorithms and thus boosts the performance from the computational viewpoint.

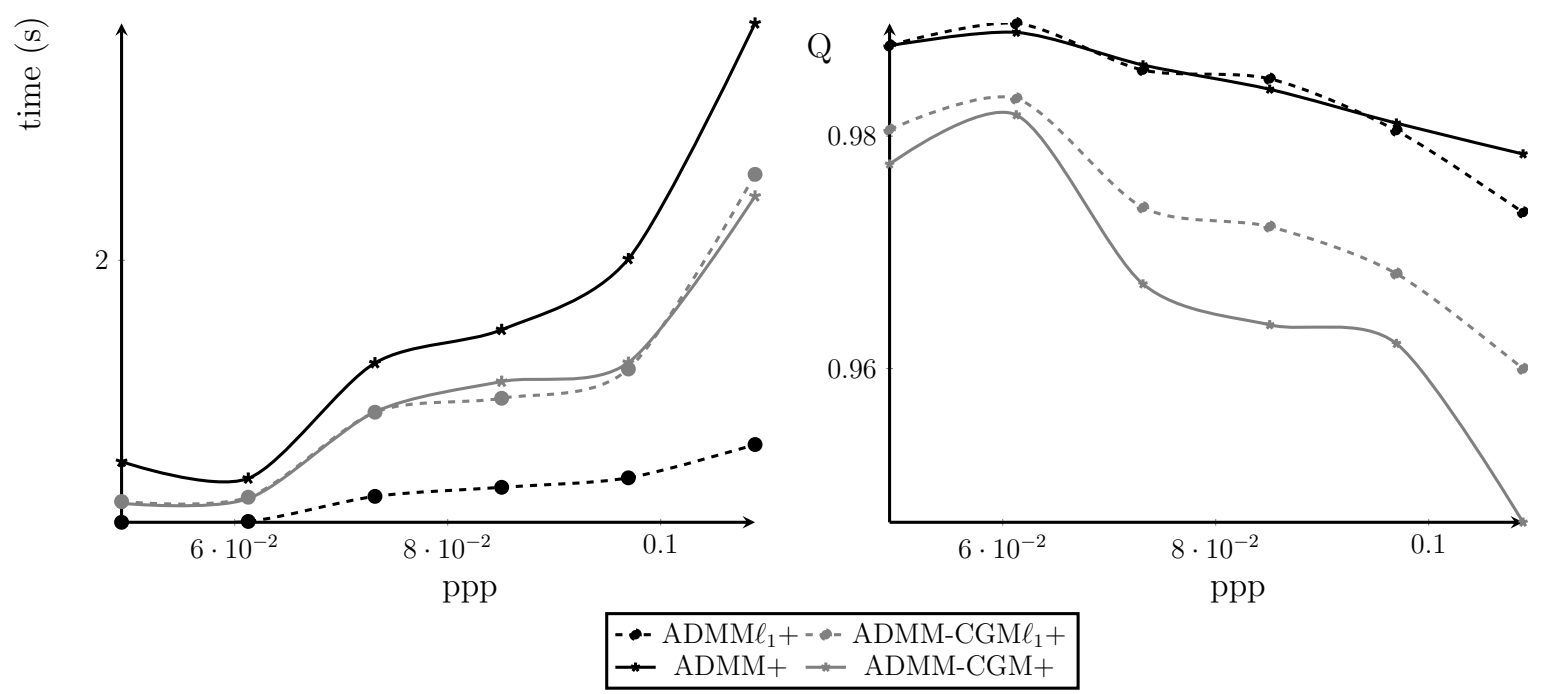

Figure 4: Left: Running time for 30 iterations against the ppp ratio. Right: Numerical assessment of the reconstruction quality of $\mathbf{w}^{\star}$ in terms of the normalized correlation against the ppp ratio. 


\section{Conclusion}

The paper tackles the crucial problem of the volume reconstruction of the particles' density at each time step of the TomoPIV chain analysis. We have presented an alternative to the "Row-Action Methods", which have been broadly employed in the literature despite suffering from a certain number of caveats. In particular, we have shown that the TomoPIV problem can be recast within a general optimization framework and that the powerful "Alternating Directions Method of Multipliers" can be used to solve the arising problem. First, we have put forth that both the physical constraints (sparsity/non-negativity) on the signal and the noise level affecting the observations can be explicitly handled by defining a proper optimization problem. Then, we have reported on a variant of the ADMM procedure based on the "Conjugate-Gradient Method" which possesses the same complexity per iteration as the "Row-Action Methods", but exhibits a faster empirical speed of convergence.

Our motivation for adapting the ADMM framework to the TomoPIV scene was driven by the nature of the problem itself. In fact, the TomoPIV volume reconstruction translates into a large-scale problem; this leads to finding an estimator built on an good trade-off between the computational load and the accuracy. ADMM has been shown to meet the latter specifications in our numerical assessment. Tests have empirically demonstrated that reconstructions are carried-out with good accuracy in only a few iterations, with, for the ADMM-CGM variant, a complexity similar to the row-action methods. State-of-the-art SMART is systematically outperformed by the newly introduced algorithmic framework. The ADMM performance in terms of the quality of the reconstruction is akin to that of the accelerated counterpart of SMART (A-SMART), with some reservations. In point of fact, the A-SMART is prone to top the $\mathrm{ADMM}$ if we let the former run up until convergence; however, for a value of maximum iterations commonly used in the experimental scene, the latter outruns its competitor. Moreover, the ADMM is easy to tune, as the only input parameter accounts for the level of noise on the observations and it is thus driven by a physical intuition. This is not the case for (A)-SMART, which is highly depended on the relaxation parameter.

The attractive properties of the ADMM approach concerning both algorithmic and computational aspects deserve further study. Our future work will take a closer look at variants of ADMM in connection with the constraints of the TomoPIV system. For example, we have noticed, in [4, Section 3.10], that the proximal methods with the Kullback-Leibler (KL) divergence can outperform the same methods with the quadratic term. We will take a closer look at variants of ADMM replacing the quadratic penalty term in problems (8)-(9) by the KL distance, in the line of work done by Wang et Banerjee in [43]. From a practical viewpoint, we will address the implementation of ADMM in a distributed computing environment; for more details, see [9, Section 10]. This could give an additional speed-up to our method. Finally, we believe that the robustness of ADMM with respect to different parameters of a real scene is to be verified by intensive experiments on real data; future work will focus on the ingress 
of the ADMM into the TomoPIV experimental scene.

\section{Acknowlegdements}

The authors thank the French Agence Nationale de la Recherche (ANR) which has partially supported this research through the "BECOSE" project.

\section{Appendix A. The Derivation of the Direct Model $\mathrm{y}=\mathrm{Ax}$}

\section{The TomoPIV Model}

Let $w(\mathbf{k})$ be the particle density at a position in space $\mathbf{k} \in \mathbb{R}^{3}$. Then, the $3 \mathrm{D}$ signal simultaneously projects onto the camera screens such that each pixel represents the integration of the 3D intensity function along a cone of view $\Omega(\mathbf{u})$ emerging from the optical center of the camera and passing through the pixel located at $\mathbf{u}$, that is:

$$
y(\mathbf{u})=\int_{\Omega(\mathbf{u})} w(\mathbf{k}) \mathrm{d} \mathbf{k},
$$

In its matrix form, Equation (A.1) writes:

$$
\mathbf{y}=\mathbf{B w}
$$

where $\mathbf{y}($ resp. $\mathbf{w})$ is the vector obtained by collecting the values of $y(\mathbf{u})$ (resp. $w(\mathbf{k}))$ on some pixel grid $\mathcal{U}=\left\{\mathbf{u}_{1}, \ldots, \mathbf{u}_{m}\right\}$ (resp. voxel grid $\tilde{\mathcal{K}}=\left\{\tilde{\mathbf{k}}_{1}, \ldots, \tilde{\mathbf{k}}_{\tilde{n}}\right\}$ ). The definition of matrix $\mathbf{B} \in \mathbb{R}^{m \times \tilde{n}}$ results from a numerical approximation of the integral in (A.1) based on a subvoxel sampling scheme inspired by [42].

\section{The Hybrid Model}

As mentioned in Section 3.1, when a seeding particle of such trivial physical dimensions is projected onto the camera screen, it impacts an aggregate of pixels. In order to mimic the image formation of the particle projections, we enforce a particular model on the physical density function $w(\mathbf{k})$ :

$$
w(\mathbf{k})=\sum_{i=1}^{n} g\left(\mathbf{k}-\mathbf{k}_{i}\right) x_{i},
$$

where $\mathbf{k}_{i}$ takes on values on a subvoxel grid $\mathcal{K}=\left\{\mathbf{k}_{1}, \ldots, \mathbf{k}_{n}\right\}$ and $g(\mathbf{k})$ is a Gaussian function with a standard deviation of 0.8. In its matrix form, Equation (A.3) reads:

$$
\mathbf{w}=\mathbf{G x}
$$

where $\mathbf{G} \in \mathbb{R}^{\tilde{n} \times n}$ such that $g_{i j}=g\left(\tilde{\mathbf{k}}_{i}-\mathbf{k}_{j}\right), \tilde{\mathbf{k}}_{i} \in \tilde{\mathcal{K}}, \mathbf{k}_{j} \in \mathcal{K}$ and $\mathbf{x}$ collects the $x_{i}$ coefficients. Plugging model (A.4) into the image projection equation (A.2) leads to:

$$
\mathbf{y}=\mathbf{B G x} \text {. }
$$

We notice that Equation (A.5) is equivalent to model (2) by setting $\mathbf{A}=\mathbf{B G}$. 


\section{References}

[1] M.V. Alfonso, J.M. Bioucas-Dias, and M.A. Figueiredo. An Augmented Lagrangian Approach to the Constrained Optimization Formulation of Imaging Inverse Problems. IEEE Trans Image Process, 20(3), 2011.

[2] A.H. Andersen and A.C. Kak. Simultaneous Algebraic Reconstruction Technique (SART): a superior implementation of the ART algorithm. Ultasonic Imaging, 6, 1984.

[3] C. H. Atkinson and J. Soria. An efficient simultaneous reconstruction technique for tomographic particle image velocimetry. Exp. Fluids, 47(4-5), 2009.

[4] I. Barbu. Tridimensional Estimation of Turbulent Fluid Velocity. PhD thesis, Université Rennes $1,2014$.

[5] I. Barbu and C. Herzet. Accelerated, Sparsity-Aware Generalizations of Classical Algorithms for TomoPIV. In Proc. PIV, 2015.

[6] I. Barbu, C. Herzet, and E. Mémin. Sparse models and pursuit algorithms for PIV tomography. In FVR, 2011.

[7] I. Barbu, C. Herzet, and É. Mémin. Joint Estimation of Volume and Velocity in TomoPIV. In PIV, 2013.

[8] C.F. Bohren and D.R. Huffman. Absorption and Scattering of Light by Small Particles. A WileyInterscience Publication, 1983.

[9] S.P. Boyd, N. Parikh, E. Chu, B. Peleato, and J. Eckstein. Distributed Optimization and Statistical Learning via the Alternating Direction Method of Multipliers. Found. Trends Mach. Learning, 3(1), 2011.

[10] C.L. Byrne. Iterative Image Reconstruction Algorithms Based on Cross-Entropy Minimization. IEEE Trans. Image Process., 2(1), 1993.

[11] Y. A. Censor and S. A. Zenios. Parallel Optimization: Theory, Algorithms and Applications. Oxford University Press, 1997.

[12] F. Champagnat, P. Cornic, A. Cheminet, B. Leclaire, G. Le Besnerais, and A. Plyer. Tomographic PIV: particles vs blobs. Meas. Sci. Technol., 2014.

[13] G. Cimmino. Calcolo approsimato per le soluzioni dei sistemi di equazioni lineari. La Ric. Sci., 14(2), 1938.

[14] P. Cornic, F. Champagnat, A. Cheminet, B. Leclaire, and G. Besnerais. Computationally efficient sparse algorithms for tomographic PIV Reconstruction. In PIV, 2013.

[15] W. Dai and O. Milenkovic. Subspace Pursuit for Compressive Sensing: Closing the Gap Between Performance and Complexity. CoRR, 2008.

[16] J. Eckstein and D.P. Bertsekas. On the Douglas-Rachford Splitting Method and the Proximal Point Algorithm for Maximal Monotone Operators. Math. Prog., 55(3), 1992.

[17] T. Elfving. On Some Methods for Entropy Maximization and Matrix Scaling. Linear Algebra Appl., 34(12), 1980.

[18] T. Elfving, T. Nikazad, and C. Hansen. Semi-convergence and relaxation parameters for a class of SIRT algorithms. SIAM J. Imaging Sci., submitted.

[19] G. Elsinga, F. Scarano, B. Wieneke, and B. van Oudheusden. Tomographic particle image velocimetry. Exp. Fluids, 41(6), 2006.

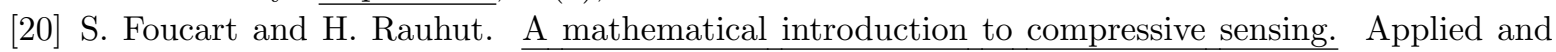
Numerical Harmonic Analysis. Birkhaüser, 2013.

[21] S. Gesemann, D. Schanz, A. Schröder, S. Petra, and C. Schnörr. Recasting Tomo-PIV reconstruction as constrained and L1-regularized nonlinear least squares problem. In SALTFM, 2010 .

[22] G. T. Herman and A. Lent. Iterative reconstruction algorithms. Comput. Biol. Med., 6(4), 1976.

[23] C. Herzet and A. Drémeau. Bayesian Pursuit Algorithms. In EUSIPCO, 2010.

[24] M. Hong and Z-Q. Luo. On the Linear Convergence of the Alternating Direction Method of Multipliers. arXiv:1208.3922. 
[25] S. Kaczmarz. Angenäherte Aufösung von Systemen Linearer Gleichungen. J. Theor. Biol, 35, 1937.

[26] R. Meynart. Digital image processing for speckle flow velocimetry. Rev. Sci. Instrum., 29(35), 1982.

[27] D. Needell and J.A. Tropp. CoSaMP :Iterative signal recovery from incomplete and inaccurate sample. Appl. Comput. Harmon. Anal., 26(3), 2009.

[28] Y. Nesterov. A method for solving the convex programming problem with convergence rate $\mathcal{O}(\sqrt{(}(k))$. Dokl. Akad. Nauk. SSSR, 269, 1986.

[29] N. Parikh and S.P. Boyd. Proximal Algorithms. Found. Trends Optim., 2013.

[30] Y. C. Pati, R. Rezaiifar, and P. S. Krishnaprasad. Orthogonal Matching Pursuit: Recursive Function Approximation with Applications to Wavelet Decomposition. In ASILOMAR, 1993.

[31] S. Petra, C. Popa, and C. Schnörr. Enhancing Sparsity by Constraining Strategies: Constrained SIRT versus Spectral Projected Gradient Methods. In VMM, 2008.

[32] S. Petra, C. Popa, and C. Schnörr. Extended and Constrained Cimmino-type Algorithms with Applications in Tomographic Image Reconstruciton. Int. J. Comput. Math., 2010.

[33] S. Petra, C. Schnörr, F. Becker, and F. Lenzen. B-SMART: Bregman-Based First-Order Algorithms for Non-negative Compressed Sensing Problems. In SSVM, 2013.

[34] S. Petra, C. Schnörr, A. Schröder, and B. Wieneke. Tomographic Image Reconstruction in Experimental Fluid Dynamics: Synopsis and Problems. In WMM, 2007.

[35] S. Petra and Schnörr, C. TomoPIV meets Compressed Sensing. Pure Math. Appl., 2009.

[36] S. Petra, A. Schröder, and C. Schnörr. 3D Tomography from Few Projections in Experimental Fluid Mechanics. In Nitsche, W. and Dobriloff, C., editor, Imaging Measurement Methods for Flow Analysis, volume 106. Springer, 2009.

[37] S. Petra, A. Schröder, B. Wieneke, and C. Schnörr. Tomography from Few Projections in Experimental Fluid Dynamics. In Imaging Measurement Methods for Flow Analysis, 2009.

[38] W.H. Press, S.A. Teukolsky, W.T. Vetterling, and B.P. Flannery. Numerical Recipes in C: The Art of Scientific Computing (second edition). Cambridge University England EPress, 1992.

[39] D. Schanz, S. Gesemann, A. Schroder, B. Wieneke, and D. Michaelis. Tomographic reconstruction with non-uniform optical transfert functions (OTF). In SALTFM, 2010.

[40] D. Schanz, S. Gesemann, A. Schröder, B. Wieneke, and M. Novara. Non-uniform optical transfer functions in particle imaging: calibration and application to Tomographic reconstruction. Meas. Sci. Technol., 24(2), 2013.

[41] J.R. Shewchuk. An Introduction to the Conjugate Gradient Method Without the Agonizing Pain. Technical report, School of Computer Science, Carnegie Mellon University, 1994.

[42] L. Thomas, R. Vernet, B. Tremblais, and L. David. Influence of geometric parameters and image preprocessing on tomo-piv results. In SALTFM, 2010.

[43] H. Wang and A. Banerjee. Bregman alternating direction method of multipliers. In Advances in Neural Information Processing Systems 27. Curran Associates, Inc., 2014.

[44] B. Wieneke. Iterative reconstruction of volumetric particle distribution. Meas. Sci. Technol., 24(2), 2013.

[45] N. A. Worth and T. B. Nickels. Acceleration of Tomo-PIV by estimating the initial volume intensity distribution. Exp. Fluids, 45(5), 2008. 